\title{
Carnets
}

Revue électronique d'études françaises de l'APEF

Deuxième série - 5 | 2015

Imaginaires de guerre et autres conflits

\section{Jeunes écrivains durant la première occupation allemande de la Belgique}

\section{Marc Quaghebeur}

\section{(2) OpenEdition}

1 Journals

\section{Édition électronique}

URL : http://journals.openedition.org/carnets/293

DOI : $10.4000 /$ carnets.293

ISSN : 1646-7698

Éditeur

APEF

\section{Référence électronique}

Marc Quaghebeur, " Jeunes écrivains durant la première occupation allemande de la Belgique », Carnets [En ligne], Deuxième série - 5 | 2015, mis en ligne le 30 novembre 2015, consulté le 05 mai 2019. URL : http://journals.openedition.org/carnets/293 ; DOI : 10.4000/carnets.293

Ce document a été généré automatiquement le 5 mai 2019.

\section{(c) (i) (8)}

Carnets est mis à disposition selon les termes de la licence Creative Commons - Atribution - Pas d'utilisation commerciale 4.0 International. 


\title{
Jeunes écrivains durant la première occupation allemande de la Belgique
}

\author{
Marc Quaghebeur
}

1 La prise en compte des traces et des effets du premier conflit mondial sur les lettres belges francophones constitue un sujet vaste et complexe. Ils découlent en effet de situations bien différentes (Front de l'Yser; Front d'Afrique centrale ; Belgique occupée ; déportation en Allemagne ; camps aux Pays-Bas; vie en France ou en Grande-Bretagne). Ils concernent en outre des générations littéraires bien différentes. Cet article se penche sur les attitudes et/ou devenirs de quelques jeunes écrivains demeurés en pays occupé, et qui marqueront les années à venir. Certains d'entre eux choisirent de ne pas s'en tenir au silence civique qui fut de mise pour beaucoup en signe de résistance. Tous ont été marqués peu ou prou par les événements révolutionnaires de 1917 en Russie. Le cas de Clément Pansaers constitue l'exemple le plus frappant et le plus ambigu de comportements littéraires actifs, voire activistes, dans un pays rudement mis à mal.

2 L'occupation de l'essentiel du territoire de la Belgique par les troupes de l'empereur Guillaume II avait en effet durement frappé les masses ouvrières et paysannes. Elle avait mis à mal le mode de vie de la bourgeoisie. Elle avait entraîné sur le continent, qui affirmait apporter la civilisation aux peuples colonisés, un déchaînement de violence tout à l'opposé, et fait s'interroger les uns et les autres sur ce qu'il en était de l'humanisme. Elle avait provoqué en outre la révolution russe - et donc, la remise en cause de tout l'édifice social et l'accouchement d'une grande part du $\mathrm{xx}^{\mathrm{e}}$ siècle. Elle avait d'autre part conforté autour de la Belgique la grande génération léopoldienne des Verhaeren, Maeterlinck ou Destrée mais entraîné divers clivages dans le champ littéraire belge francophone en devenir. Si d'aucuns se trouvent aux tranchées, comme Lucien Christophe ou Max Deauville, la majeure partie des écrivains vit en effet en pays occupé.

La plupart des écrivains demeurés en Belgique occupée publient peu, ou pas, par devoir civique. Ceux qui se trouvent à l'étranger, comme Verhaeren ou Maeterlinck, mènent campagne pour la Belgique martyre. Personnalités fondatrices de la grande génération léopoldienne, Edmond Picard ou Georges Eekhoud qui vivent à Bruxelles confient à leurs journaux, non seulement des traces de leur quotidien, des descriptions des attitudes 
affairistes de certains de leurs compatriotes, mais aussi les débats qu'entraînent en eux les contradictions issues de leur pacifisme et de leur patriotisme, de leur hantise du monde culturel germanique et de la réalité de l'occupation allemande, de leur passion de la littérature et de l'Art, et du statut qu'ils sont censés occuper en conséquence ${ }^{1}$.

\section{Des liens avec la littérature allemande contemporaine}

4 D'autres, plus jeunes, se retrouvent parfois, en pays soumis, au contact de grands écrivains allemands qui vivent alors en Belgique ${ }^{2}$, tel Carl Sternheim. Ils se frottent ainsi à une modernité différente de celle, plus ancienne, de Verhaeren ou de Maeterlinck. Cette modernité ne va pas forcément au bout du questionnement du langage mais rebat les cartes d'un horizon culturel dans lequel Mallarmé, Rimbaud ou Flaubert continuent de jouer également un rôle.

5 Pour qui ne se l'interdit pas par devoir patriotique, une vie culturelle existe donc malgré l'occupation, voire à travers elle. Elle amène ainsi René Verboom, Clément Pansaers ou Michel de Ghelderode à se retrouver au Compas, café du bas de la ville de Bruxelles où passe également Fernand Crommelynck, lequel ${ }^{3}$ met même sur pied, en 1916, un «Théâtre volant » qui a du succès. Verboom et Ghelderode se retrouvent également à La Hulpe chez Clément Pansaers, devenu secrétaire de l'écrivain allemand Carl Sternheim, et précepteur de ses enfants. Ils se frottent ainsi à d'autres enjeux que ceux de leur mémoire culturelle nationale et française.

6 Future figure-clé du dadaïsme en Belgique dans l'immédiat après-guerre, Clément Pansaers (1885-1922) publie, entre 1917 et 1918, des Cahiers mensuels littéraires illustrés, sous le titre Résurrection ${ }^{4}$. Namur en est le lieu d'édition - l'adresse de la rédaction étant à La Hulpe. Vivant de l'aide de Berlin, cette revue d'esprit pacifiste et de sensibilité expressionniste tempérée est porteuse de velléités révolutionnaires. Elle publie des textes de Charles Vildrac ou de Pierre-Jean Jouve mais aussi d'Alfred Wolfenstein ou Herwarth Walden. Elle débute par une longue étude de la nouvelle littérature allemande rédigée par Pansaers, étude qu'il ne faut pas analyser pour sa précision mais retenir en fonction de l'esthétique personnelle qui s'y cherche, du contexte de parution et des nombreux jeux de renvoi aux auteurs français qu'elle comporte tout autant. Fille de la simplicité, écrit ainsi Pansaers, la France produit Taine qui « simplifie Hegel $»^{5}$ (Pansaers, Résurrection, 1, 1917 : 7). La revue rend hommage à Frank Wedekind, qui vient de décéder, mais publie également un premier autoportrait fantasmatique du jeune Ghelderode. Elle ne peut être considérée comme une revue d'avant-garde au sens strict. À côté d'Ywan Goll et de Carl Einstein, que Pansaers traduit, on trouve par exemple la signature de Pierre Benoît.

7 Si la revue ouvre en revanche à René Verboom ou à Michel de Ghelderode les fenêtres de belles et premières publications, celles-ci ne brillent pas, comme d'autres d'ailleurs qu'accueille la revue, par leur modernisme ${ }^{6}$. Verboom commence ainsi son poème «Les plus doux» par un alexandrin ronflant presque verhaerenien, mais significatif du contexte auquel il s'oppose : "Au fond d'un siècle mâle héroïsant la force » (Verboom, Résurrection, 4, 1918: 155). Pansaers lui-même ne déroge pas toujours à ce type de filiation : « Et tant qui meurent sans amour ! / Voici que l'homme adore la mort. // - 0 la beauté de la vie forgiaque ! - (Pansaers, Résurrection, 4, 1918:136) L'on est donc loin du dadaïsme radical du Pansaers des années 1919-1921. L'emprise expressionniste du poète « national » décédé l'année précédente s'y lit même. 


\section{L'hymne à la Vie et l'analyse politique sous censure allemande}

8 La revue entend toutefois faire bouger les lignes - et pas seulement en matière esthétique, domaine dans lequel la mise en cause du siècle écoulé est patente mais continue à renvoyer à l'Esprit. Elle le proclame. L'Appel à l'Art d'Ywan Goll invite ainsi le poète à ne pas avoir

honte de souffler dans la trompette. Viens avec la révolte. Roule le tonnerre dans les minces nuages de la rêverie romantique, jette l'éclair de l'esprit dans la masse. Cesse les tromperies doucereuses et les légers désespoirs des pluies et des fleurs au crépuscule. [...] Chante des hymnes, crie des manifestes, forge des programmes pour le ciel et la terre. Pour l'esprit ! (Goll, Résurrection, 4, $1918: 122$ )

Évident dans son intention mais encore empêtré dans une rhétorique que le surréaliste Paul Nougé balayera à partir de 1924, le projet de Résurrection se veut bien évidemment novateur : en phase avec la Vie; en exaltation « dans l'humain » pour paraphraser Ywan Goll (Goll, Résurrection, 4, 1918 : 130). Pour Pansaers - en quoi il est plus proche qu'il ne le dit de certains de ses adversaires - l'artiste est celui qui permet de s'élever et de dépasser «la satisfaction du ventre» (Pansaers, Résurrection, 6, 1918: 207). Son œuvre « doit nous aider à vivre » - ce qui «ne signifie pas que l'art doit être spécifiquement social.» (Pansaers, Résurrection, 6, 1918: 208) Pour preuve, Mallarmé ou Baudelaire que le jeune directeur de revue qualifie de «statiques » (Pansaers, Résurrection, 6, 1918 : 209).

L'articulation, ou plutôt la juxtaposition du littéraire et du politique dans la revue, en découle donc logiquement. Elle est différente de ce qu'elle sera dans les avant-gardes à venir. D'un côté, le moraliste écrit que

[1 ]es habitudes ont tellement maquillé la vie, que la plupart des humains sont encore incapables de la fixer toute nue et de l'embrasser ainsi de tous leurs sens. Et dans l'erreur, comme en toute valeur, il y a des degrés, dont un - le plus important peutêtre - est la sentimentalité. $»^{7}$ (Pansaers, Résurrection, 6, 1918 : 210)

De l'autre, dans chaque numéro de cette revue littéraire très singulière puisqu'elle paraît sous censure allemande - cas de figure rarissime du côté belge francophone -, Clément Pansaers publie des billets politiques.

Ceux-ci donnent une vision, presque unique à l'époque, et en français, de la situation de la Belgique et surtout de son évolution. Ses perspectives sont en effet à l'exact opposé de ce que pensent ceux qui vont libérer le pays du joug allemand, comme de celles de la plupart des Belges survivant sous la botte allemande. Les billets de Pansaers semblent, qui plus est, peu articulés à la partie littéraire et picturale de la revue. Ils correspondent en revanche au titre Résurrection puisqu'ils entendent déclencher un autre aspect de la révolte, plus politico-social. Ils le font au moment où Berlin, que dirige de plus en plus l'État-major, a décidé d'exploiter plus que jamais les ressources de la Belgique; d'accélérer le processus de séparation du Nord et du Sud du pays; et, si possible - du moins pour un certain nombre de cercles influents de la capitale impériale -, d'aboutir à l'annexion à l'empire de tout ou partie du royaume. 


\section{Les prospectives révolutionnaires tous azimuts de Clément Pansaers}

13 Impossible de ne pas tenir compte aussi de tels paramètres et de se contenter d'une lecture purement littéraire de Résurrection. Comme l'indication "Namur», le «Bulletin politique » de chaque numéro inscrit le périodique dans la logique de la Flamenpolitik, et de son pendant wallon. Aucun doute 8 à avoir sur la stratégie allemande par rapport à Résurrection - ce qui n'autorise pas à s'y limiter pour commenter jeux et enjeux de cette revue. La perception de l'importance historique de ce périodique dans l'histoire des lettres belges s'accrut d'ailleurs tardivement - et ce n'est pas un hasard -avec la prise en compte, dans les années 1970-1980, de l'histoire des avant-gardes belges par-delà le silence accablant de l'histoire littéraire officielle à leur égard et l'éloignement progressif de la mémoire de 1914-1918. Cela amena le renouveau critique à se préoccuper assez peu de l'aspect politique ambigu de l'aventure de Résurrection.

L'espoir révolutionnaire suscité par les événements de Russie complique en outre la lecture et l'analyse. Octobre 1917 exerce bien sûr un effet détonant sur Pansaers qui ne se contente pas de démasquer le jeu capitaliste du président américain Thomas Woodrow Wilson. Elle lui fait entrevoir la possibilité d'une tabula rasa sociale qui irait de pair avec une évolution institutionnelle radicale de la Belgique. La position du commentateur à l'égard de l'évolution du pays et des formes de résolution de la question des langues ${ }^{9} \mathrm{a}$ quelque chose de prospectif, voire de prophétique, comme je l'indiquais dès 1982 dans mes «Balises» (Quaghebeur, 1982: 65-70). Pansaers parle en effet de fédération entre Flandre et Wallonie - mais dans le sens confédéral de deux États. Il inscrit Bruxelles en Flandre, ce qu'a toujours revendiqué le flamingantisme. Symbole de la Belgique que Pansaers veut voir disparaître, et de sa complexité, la capitale de "l'ancien Etat " (Pansaers, Résurrection, 2, 1918: 78) ne pèse pas lourd pour lui. Le chroniqueur et polémiste propose ainsi de donner au futuriste italien Marinetti - il parait visiblement le hanter - une belle part des collections muséales de la capitale.

Le moment choisi par Pansaers pour de telles assertions est celui où la pression allemande sur la Belgique est maximale. Plus d'un demi-million d'hommes sont sans travail du fait de l'occupation. Cela fait d'autant plus question que Pansaers traite, en temps de guerre, ceux qui défendent une opinion autre que la sienne - opinions de fait anti-allemandes avec une belle mauvaise foi et une violence rare. Pansaers ne semble pourtant pas aveugle au contexte, lui qui écrit, dans le cinquième Bulletin, "nous agissons par opportunité " (Pansaers, Résurrection, 5, 1918: 196). Il vient de se déchaîner contre les huit figures de proue du mouvement flamand (dont August Vermeylen ou Herman Teirlinck). Ceux-ci ont écrit au chancelier de l'empire allemand une lettre attestant leur patriotisme belge et leur refus de collaborer aux desseins allemands, même pour défendre la cause flamande. Une telle prise en compte de la Belgique transforme les signataires en traîtres et en vieilles lunes.

Largement répandue - ce qui explique la blessure ultérieure de membres du mouvement flamand -, la position des militants flamands non activistes peut être rapprochée, toutes proportions gardées, de celle de Jules Destrée auquel Pansaers n'a pas manqué de réserver des flèches dès son premier Bulletin. En s'en prenant aux huit personnalités flamandes engagées dans le combat flamand comme à la figure de proue du mouvement wallon qui plaide partout dans le monde, depuis 1914, la cause de la Belgique, Pansaers 
sert de fait la politique de l'occupant. Il le fait avec violence et simplisme, assimilant à de la lâcheté et à une fuite la présence en août 1914 du député de Charleroi qu'est Destrée hors de son canton électoral, puis à l'étranger. Avant de devenir le propagateur de la cause de son pays à l'étranger, Destrée avait en effet suivi la retraite de l'armée, du gouvernement, et du Parlement dont il était membre.

Dans son Bulletin et dans cette logique, Pansaers affirme que les représentants politiques élus avant 1914 - et donc le gouvernement en exil (à Sainte-Adresse) qui en procède n'ont plus aucune légitimité. Les émigrés sont « devenus de parfaits étrangers » au pays. Ils sont en outre considérés comme la quintessence de ce que Pansaers dénigre ou vomit. Le Belge a-t-il jamais été capable de "penser par lui-même? (Pansaers, Résurrection, 4, 1918 : 159) Certes avec de tout autres circonvolutions, ce type de mépris à l'égard de ses compatriotes se retrouve plus tard chez maints signataires du Manifeste du lundi. Il plonge dans la difficulté ou l'impossibilité de prendre en compte la différence belge. Celle-ci équivaut en sus pour Pansaers au verrou qui empêcherait la révolution sociale.

Dans toutes ses chroniques, Pansaers se garde de parler des soldats qui combattent derrière l'Yser. Encore plus, de ceux qui se sont battus en Afrique centrale où ils ont défait les troupes allemandes. La qualification des Belges occupés est par ailleurs totalement dépourvue d'apprêt, voire de compréhension : «Ignorant ce qu'on pense audelà des tranchées, on en est encore ici à la haine nocive " de l'Allemand (Pansaers, Résurrection, 4, 1918 : 159). C'est qu'il ne s'agit surtout pas de suivre « ceux - flamands ou wallons - qui prétendent dédoubler la petite vérité belge périmée.» (Pansaers, Résurrection, 3, 1918: 119) Pour y atteindre, il s'agit en revanche de mobiliser les masses en évitant leur contamination par l'esprit de l'Yser. Et de pouvoir agir, ce faisant, à l'instar de ce qui s'est produit en Russie ${ }^{10}$ : «Juridiquement comme d'après toute saine logique seul, l'élément de la population resté dans le territoire - la forte majorité d'ailleurs - qui représente et constitue le peuple a, en toute éventualité, à se prononcer et à prendre des décisions. » (Pansaers, Résurrection, 6, $1918: 237)^{11}$ Ce peuple, c'est bien évidemment aux élites éclairées, auxquelles appartient le chroniqueur et analyste engagé, que revient le rôle de lui apprendre le comportement adéquat.

En revanche, le refus de la théorie de la «race» (Pansaers, Résurrection, 3, 1918: 119) comme le mélange de bolchévisme et de pacifisme sont évidents chez Pansaers. Ils vont de pair avec un aveuglement relatif ${ }^{12}$ sur le jeu de Berlin. Ou sur une adhésion à celui-ci, comme tremplin? Ils passent en tous les cas par le mépris de tout autre position que la sienne sur la Belgique, mépris qui confine souvent à la haine et à la rage. Typique en un sens des élites belges - particulièrement dans les décennies qui vont suivre --, ce mépris de Soi dont Michel de Ghelderode donnera plus tard une image particulièrement ambiguë et souvent grotesque, s'inscrit chez Pansaers dans un discours de lutte contre ce que la Belgique d'avant 1914 et celle du Front de l'Yser sont censées représenter. Il n'en reproduit pas moins un fantasme d'absorption, hanté par la projection dans l'autre - qu'il fût russe, allemand ou français.

À l'effondrement du mythe germano-latin et de l'Idéal qui caractérise les réactions du monde littéraire belge à l'invasion d'août 1914 et à la violation par l'Allemagne de la neutralité de la Belgique, dont elle était garante, Pansaers répond par un réinvestissement de la culture germanique contemporaine et une ouverture aux auteurs français unanimistes. Politiquement, il le fait par la défense d'une Wallonie à créer, sans aller très loin dans ses réflexions à l'égard de l'emprise allemande, et dans un contexte d'occupation qui donne à son propos une résonance différente de celle d'opinions 
wallonnes autonomistes qui se liront chez certains écrivains des tranchées, tel Louis Boumal. Comparant les contradictions internes qui l'agitent ainsi que l'humanité à un "champ de bataille», il se contente de rappeler l'abrutissement auquel a mené le « raffinement d'hier ». Il se dit convaincu que les années de guerre vont déboucher sur la « confraternité des nations » (Pansaers, Résurrection, 1, 1917 : 37), rêve qui était celui de la génération léopoldienne avant le séisme d'août 1914, il faut le rappeler. Comme chez tous, la question du "monde d'hier », pour paraphraser Stefan Zweig, est donc bel et bien présente.

21 Pansaers en tire en outre des conclusions au niveau francophone belge, qu'il réduit à sa composante wallonne. Et d'estimer que « le wallon a le devoir de se prononcer et de se défendre » - «le flamand ayant résolu le problème de son identité ». Pansaers se dit convaincu que l'Allemagne - « ne désire pas annexer la Belgique » (Pansaers, Résurrection, $1,1917: 39)^{13}$, credo qui est pourtant celui d'une part influente des élites militaires et économiques de l'empire. Dans son projet, Pansaers achoppe bien évidemment sur le problème de Bruxelles auquel il répond en se référant notamment à Baudelaire - ce qui prolonge, là encore, une tradition littéraire d'autodénigrement que masque mal le besoin du grand Autre. Les termes choisis par Pansaers sont particulièrement significatifs d'une hantise de l'homogène chez le jeune révolutionnaire en herbe :

Les Wallons regrettent un centre! Mais quel centre? ---Amphibie, baroque, hybride. Rappelez-vous ce que Baudelaire, mais surtout Octave Mirbeau en a dit ! Et puis, le Wallon n'a-t-il pas l'avantage de pouvoir édifier d'un bloc un milieu adéquat à son temps, à son tempérament ? (Pansaers, Résurrection, 2, 1918: 78) ${ }^{14}$

\section{Affirmation plus que création d'une esthétique nouvelle}

La pensée de Pansaers, comme sa vision du littéraire, n'est toutefois pas dépourvue, ellemême, d'hybridité. Son propos se double non seulement de la dimension révolutionnaire ouverte par les Soviets mais aussi de la reprise de ce que portait une part de l'esprit du $\mathrm{XIX}^{\mathrm{e}}$ siècle en Belgique, et que le patriotisme risquait en effet de recouvrir.

Soyons travailleurs comme nos mineurs, nos métallurgistes. Notre peuple nous apporte son industrie, donnons-lui une culture de valeur cosmopolite. Faisons de la Wallonnie (sic) ce que la Belgique aurait dû réaliser --- un boulevard international, où toutes les races se croisent et se mesurent confraternellement dans le domaine intellectuel et artistique. (Pansaers, Résurrection, 2, $1918: 79$ )

C'est bien évidemment vers ce renouveau artistique, concomitant de l'émancipation du prolétariat, que se tourne un écrivain soucieux d'exalter «la vie »; de dépasser le temps où l'artiste équivalait à un " charlatan licencié ès-intrigues (sic) » (Pansaers, Résurrection, 2, $1918: 79)$; et de se forger, au firmament littéraire, une place capitale. Dans ce contexte, Pansaers réfléchit à la poétique des temps nouveaux qu'il appelle de ses vœux. Il insiste sur l'inutilité pour le poète de "son jeu de vocables creux, la nullité de sa petite sensibilité devant le sublime effarant de la vie, qui se renouvelle dans les affres de la mort » (Pansaers, Résurrection, 1, $1917: 38)^{15}$ mais n'en donne pas toujours des exemples convaincants dans les choix qu'il effectue au sein de sa revue.

Bien qu'il les évoque, Pansaers ne s'attaque pas alors au mal insidieux que le premier conflit mondial a instillé dans la langue. Il y répondra en revanche, et de façon radicale, dans sa trajectoire dadaïste - après l'échec de la révolution spartakiste ${ }^{16}$. Au niveau 
littéraire, ce qu'il publie dans Résurrection révèle une sensibilité moderne mais ne va pas très loin en termes d'invention du nouveau - n'étaient sa pièce Saltimbanques et certaines traductions de l'allemand. Lui qui croit au renouveau, presque miraculeux, ne va pas alors au bout de la défiance à l'égard des Formes et des Mots qui caractérisera une part du demi-siècle, et suscitera tant des réactions passionnées, quasiment réactionnaires, de crispation sur le français.

\section{Le coup de balai social}

La disparition de la Belgique (d'hier) et la régénérescence du "petit belge » (sic) (Pansaers, Résurrection, 2, 1918: 76), à l'instar de la gestion de la révolution par les Bolchéviques $^{17}$, doit être confiée, je l'ai dit, à une élite nouvelle dont Pansaers se veut un des parangons - l'élite précédente assistant « en quiétude à la dépravation générale » et " les émigrés se trouv[a]nt vis-à-vis du problème belge comme des stratèges en chambre " (Pansaers, Résurrection, 4, $1918: 159)$. Après l'armistice de 1918, et à l'heure de la lutte entre la future U.R.S.S. et les Puissances occidentales, de tels propos seront d'autant plus inaudibles qu'ils ont été écrits sous contrôle, voire avec la complicité de l'occupant. La reconstruction de la Belgique de l'Entre-deux-guerres prendra, qui plus est, de tout autres chemins dont la complexité, les omissions et les compromis pèseront non seulement sur la vie sociale et politique, mais aussi littéraire.

Le coup de balai social auquel aspire Pansaers est aussi celui du premier Plisnier (1896-1952) qui adhère à la Troisième Internationale, en 1919, et publie en 1921 Réformisme ou Révolution chez Ça ira !, l'éditeur anversois. La revue homonyme a publié, en 1921, le numéro 16, Dada. Sa naissance, sa vie, sa mort. Dans ce numéro où l'on retrouve Ywan Goll, Francis Picabia, Ezra Pound ou Benjamin Péret, Clément Pansaers publie un article «Dada et Moi » dans lequel il affirme être devenu dadaïste en 1916 comme par inadvertance géniale puisque «le mot Dada n'était pas encore trouvé » (Pansaers, 1921: 111) et qu'il ne le découvrit qu'en 1919, à Berlin où il s'était réfugié, grâce à Carl Einstein. Il trace clairement le lien entre ces séismes esthétiques et le conflit mondial qui « coupa net la continuité de toutes les spéculations d'avant 1914. » (Pansaers, 1921: 111) Il n'y fait plus aucune allusion à la Wallonie et aux événements politiques mais se concentre sur l'artistique/humain - Dada équivalant à la mise en valeur de la «dissemblance » et de l'horreur de la grammaire normée (Pansaers, 1921 : 112). Il affirme avoir écrit entre 1916 et 1918 ses œuvres les plus novatrices dont le Pan Pan au cul du nu nègre (Pansaers, 1921 : 114) qu'il n'a toutefois pas publié dans Résurrection.

Dans son texte écrit en 1919, Plisnier - alors partisan du terme social de l'alternative revient sur cette "guerre prévue, préparée, acceptée par la bourgeoisie universelle [qui] a tué treize millions d'hommes, sains et très utiles, anémié l'Europe entière et désorganisé la production du monde.» (Plisnier, 1921: 17) Il refuse que les survivants, « oubliant le frère assassiné, la campagne (sic) épuisée et l'enfant phtysique » (Plisnier, 1921: 17) apportent une aide quelconque à la reconstruction du monde d'hier. Se référant à Spartacus et à Lénine, il en appelle au sang et à la violence, en renvoyant aux années de « rage sanglante » durant lesquelles la bourgeoisie dominante répondait aux hommes qui voulaient mettre fin au carnage : «'Si nous cessions la guerre avant la victoire, les morts sortiraient de leurs tombeaux pour crier vengeance !'« (Plisnier, 1921: 64) 
Aucune allusion spécifique à la Belgique ; aucune considération esthétique non plus dans cet opuscule politique. Plisnier est proche de La Lanterne sourde animée par Paul Vanderborght. Il publie Ève aux sept visages, qu'il reniera par la suite mais contient les traces de la propension lyrique qui sera toujours la sienne. Il n'en fait pas état. C'est également au sortir de la guerre que Paul Nougé participe à la fondation du Groupe communiste de Bruxelles, sans doute à travers son amitié pour le peintre War Van Overstraeten - lequel devait par la suite rejoindre, comme Plisnier, le christianisme ; puis passer à la collaboration. Paul Nougé (1895-1967) mettra en place, à partir de 1924 - et à la différence de Plisnier - un dispositif d'écriture parmi les plus rigoureux au sein des avantgardes européennes. Il ne cherchera pas à faire dépendre du parti communiste son esthétique mais à inscrire la révolution littéraire au sein même et à partir de ses pratiques sans la moindre illusion fusionnelle ${ }^{18}$.

$\mathrm{Au}$ sein des avant-gardes prendra en outre bientôt figure Michel Seuphor (1901-1999), jeune flamingant qui, à la différence de Pansaers, n'accepta aucune collaboration avec les Allemands. Dès l'Armistice, il s'engage en revanche pour la pleine reconnaissance du flamand. En 1921, il crée la revue Het Overzicht qui s'approche des avant-gardes et s'écarte des idées de surhomme et de germanité chères aux activistes flamands. Dans le premier numéro de sa revue, il publie, sous son patronyme (Fernand Berckelaers), un « AntiNietzsche » qui vante l'humain, catégorie qui hante décidément les uns et les autres, n'étant Nougé. Il le fait toutefois peu explicitement. Ainsi Seuphor entame-t-il le parcours qui l'amènera à rencontrer Marinetti, Balla, Schwitters, Mondrian, Le Corbusier ou Kertész; à écrire la pièce L'éphémère est éternel (1926) ; à contribuer à L'Esprit nouveau, revue qu'il anime avec Paul Dermée à partir de 1927 ; à s'acheminer peu à peu vers son esthétique ultérieure, lumineuse et dépouillée, celle de ses peintures-mots.

\section{Une fantasmatique de l'immémorial et du trivial}

30 Pas du tout marqué à gauche, Michel de Ghelderode (1898-1962) charrie, lui, tout au long de sa vie et de ses déclarations, deux visions de la Belgique. L'une n'est pas étrangère à un rejet souvent sommaire dont il est loin d'être le seul représentant ; l'autre est d'exaltation nostalgique. Plus que beaucoup, Ghelderode va en effet habiter - et certes comme un songe - l'imaginaire du mythe belge du XIX ${ }^{e}$ siècle. Au point de s'identifier de façon croissante, et parfois pathétique, à Breughelande au fur et à mesure que l'Europe basculait dans le pire, et que la Belgique se trouvait à nouveau menacée par l'Allemagne, tout en traînant dans le burlesque ou l'abject l'essentiel de ce qui en constituait la réalité à son époque.

$31 \mathrm{Au}$ cœur de ses ambivalences, abjections ou récriminations, la question du statut littéraire et de sa reconnaissance comme écrivain majeur est dite de façon plus immédiatement manifeste et individuelle que chez son ami Pansaers, chez qui elle est toutefois aisément repérable. Sous le pseudonyme de Costenoble, vocable qui se passe de commentaire, Ghelderode écrit ainsi : «Pour avoir vécu en Belgique / pays stupide et nostalgique / nous, les pauvres littérateurs, / nous irons droit chez le Seigneur / tandis que les crétins funèbres / se moucheront dans les ténèbres. ${ }^{19}$ (Costenoble, $\left.1928: 19\right)$ Le jeune écrivain s'en prend par ailleurs - et le lien vaut d'être noté - aux anciens combattants : « du trou de mon crâne naîtront des roses / j'ai six décorations je ne suis pas morose / passé le régiment je connais les hypnoses " (Costenoble, 1928: 15) ${ }^{20}$. Ainsi s'esquisse une mémoire contrastée, d'où surgiront plus tard des œuvres importantes 
dominées par le songe et l'éclat du Verbe, mais qui n'est pas sans engranger les années passées dans la proximité de Clément Pansaers, puis celles vécues à l'armée ; enfin, dans le panorama culturel et patriotique de l'après 1918.

Enrôlé sous les drapeaux, en juin 1919, Ghelderode émerge ensuite dans le champ littéraire. Notamment à travers La Renaissance d'Occident qu'anime Maurice Gauchez, un volontaire de 1914-1918. Un prix lui est attribué pour sa pièce Oude Piet. La chronique des lettres allemandes est offerte à sa plume. Il n'en rédigera qu'une, puis s'occupera de chroniques d'expositions. La présidence du Cercle de Bruxelles de La Renaissance d'Occident lui est confiée. Plusieurs de ses textes sont publiés par la maison d'édition homonyme. Ghelderode commence à trouver la voie pour ses rages comme ses contradictions : celle du burlesque d'Oude Piet. Le Cavalier bizarre - son Intruse en somme - montre bien, d'autre part, comment il engrange et rebrasse l'héritage maeterlinckien. Comme président du Cercle de la Renaissance d'Occident, il écrit des pièces folkloriques qu'il prétendra par la suite avoir confectionnées à partir de spectacles de marionnettes. Affabulation parmi d'autres, comme l'a montré Roland Beyen, chez un écrivain pour qui le mythique ou le fabulatoire l'emporte sur le réel. Tout cela n'en débouche pas moins sur la composition moderniste de La Mort du docteur Faust (1925), démystification d'un mythe majeur de la raison moderne, comme Don Juan le sera du mythe de la femme idéalisée et de son beau séducteur. Le burlesque y confine à l'abject, ce qui n'est pas sans lien avec la débâcle des valeurs consécutives au premier conflit mondial. Avant l'aventure de la traduction et de la mise en scène de ses pièces par le Vlaamsche Volkstoneel, Ghelderode n'y substitue rien pour autant. Il plonge ensuite, décisivement, dans le songe d'une Flandre plus que jamais mythique - et cela à l'heure de l'évolution linguistique et politique de la Belgique, puis de l'occupation nazie à laquelle il répond par Le soleil se couche (1943), pièce consacrée à la retraite à Yuste du plus grand prince de son pays sous l'Ancien Régime, Charles Quint.

$\mathrm{Au}$ fur et à mesure de sa relative reconnaissance littéraire, Ghelderode cherchera à gommer l'importance de la Renaissance d'Occident dans son parcours. Il le fait dans des termes qui renvoient à certains effets de 1914-1918 dont le Manifeste du lundi constitue lui aussi un prolongement. Le dramaturge ridiculise en effet Gauchez, mentor d'une des dynamiques de l'après-guerre et historien des lettres belges. En 1961, Max Deauville ne manque pas de rappeler à Ghelderode sa dette à l'égard de Gauchez, qu'il avait comparé, dans sa préface aux Roses de Chiraz (1959) du même Deauville, à « un grotesque bonhomme tenant de l'adjudant de semaine et du maquignon » (Beyen, 1921 : 188).

Deux ans plus tôt, dans une autre préface - aux Euvres complètes de Clément Pansaers cette fois -, il affirmait, à travers des phrases emplies de dénigrement pour tout ce qui n'est pas lui, que l'assemblage des trois plaquettes rééditées suffisait à faire entrer dans

la petite Histoire des Lettres Belges, à quémander l'attention des spécialistes, des nécrophores ou nécrophages. Encore faudrait-il qu'il existât une Littérature Belge. A qui s'adresser ? Celui qui en était l'ornement a rendu l'âme - l'âme belge s'entend - il n'y a guère: l'ubuesque Gauchez - un Dada lui aussi par sa cacographique incohérence, et qui résume toute l'ignominie et le grotesque de cette Littérature Chose que nous portons dans notre cœur innombrable, comme chacun sait... (Beyen, $1971: 188$ )

Dans la préface aux Roses de Chiraz, Ghelderode résumait en outre comme suit l'histoire de la Renaissance d'Occident : groupe « constitué en grande part de gens plus ou moins de lettres qui s'étaient connus au front de l'Yser, voire derrière le front, et d'aucuns dans les bons secteurs de Londres ou de Bordeaux. Mais seul comptait l'esprit guerrier, la fraternité des armes. » (Beyen, 1921 : 187) 
dans la ligne du sous-texte du Manifeste du lundi, ce type de propos indique certaines des contradictions du champ littéraire de l'après 1918, qui s'accentueront après 1945 . Se défaire de l'appartenance belge, en littérature particulièrement ; occulter peu ou prou ce qui, en littérature, procède du choc de 1914-1918; en remettre enfin sur l'autonomisation du littéraire constituent des préoccupations d'un Manifeste dont Michel de Ghelderode fut un des signataires. Les allusions à Gauchez ne désigneraint-elles pas ceux que visait le Manifeste, sans jamais les nommer? Jeune homme pauvre en pays occupé, jeune écrivain ambitieux ayant vécu dans l'orbite déconstructiviste de Clément Pansaers, Ghelderode publie, au sortir des casernes, L'Histoire comique de Keizer Karel (1922), texte qui plonge dans les traditions orales profondes du pays - burlesque inclus - et tourne déjà autour de la figure immémoriale et fondatrice de Charles Quint. Il en écrira l'assomption en 1943, à l'heure de la mise à mal du pays natal par l'Allemagne, digne héritière - comme chez Maeterlinck - de Philippe II d'Espagne. Il ne manquera pas d'y projeter un double imaginaire de ses songes d'écrivain.

\section{L'ambiguiité mais la présence de la mémoire de 1914-1918}

En 1923, Ghelderode donne en outre L'Homme sous l'uniforme, nouvelles qui renvoient à ses années de service militaire. Ces textes, qui tournent notamment autour d'un adjudant demeuré en service et adepte du train-train militaire comme de la solitude encadrée, sont l'occasion de renvoyer à telle ou telle anecdote de la guerre; de laisser libre cours à sa hantise de la mort ; mais aussi de donner à sentir un climat de société marqué par l'aprèscoup du conflit et ses impasses ou illusions. Significative, la hantise déjà repérée chez beaucoup d'autres, de l'humain. Sur la page de garde de l'exemplaire $n^{\circ} 1$ de l'édition originale du volume, Ghelderode a écrit: «D'un temps pathétique, à la recherche de l'homme [...] 》 (Ghelderode, $1978: 9$ )

Dans la première des nouvelles du recueil, Les Hommes de la classe, l'adjudant de carrière tient à un des soldats qui le quitte des propos révélateurs de la vision du monde de l'écrivain mais aussi des séquelles morales de 1914-1918:

Oui, Benoy, je crois à ce que je dis ! Et cependant, j'ai peut-être autant d'anarchie que vous dans l'âme !... Sous mon uniforme, je suis un homme, comme vous, comme tous... J'ai horreur du meurtre, je ne crois ni aux patries, ni à l'honneur, ni à l'intelligence, ni à la civilisation... Je me suis battu pour la peau... pour des causes que j'ignore, pour des buts qui ne me sont pas montrés... J'ai de l'amertume, du dégoût à revendre !... Eh bien, je vous le dis, je ne crois à rien de mieux... Nous ne serons jamais libres !... Les peuples marcheront toujours au pas... Et quand ils se croiront délivrés, il se trouvera encore quelqu'un pour les remettre au pas !... Et puis quand même la liberté serait possible, l'homme ne la mériterait pas... Il est trop mauvais !... (Ghelderode, $1978: 19-20)^{21}$

L'Homme sous l'uniforme $e^{22}$, s'il immerge donc le lecteur dans la vie de caserne de l'immédiat après-guerre, plonge également dans divers souvenirs de celle-ci. Les nouvelles ne manquent pas de tourner en dérision les exagérations rhétoriques patriotiques des années qui suivirent l'armistice ${ }^{23}$. Ainsi Mort et glorification, récit qui se passe dans un cimetière. L'on s'apprête à y réenterrer un compagnon de combat des deux comparses qui s'y promènent et qui ont quitté l'armée. L'un des deux écrit, « avec des mots quelconques, mais qu'on n'oubliait pas tant ils racontaient les hommes et leurs âmes avec vérité. » Il 
découvre "toujours quelque chose d'étrange et d'inattendu là où personne ne voyait rien. » (Ghelderode, 1978 : 59)

L'homme qu'on va réinhumer en fanfare s'appelait Smet. Un soir de guerre, on l'amène dans un baraquement sanitaire jouxtant un cimetière où l'aumônier « ne vous confessait qu'en flamand ", et où une " douzaine d'embusqués (...) vous maniaient comme de la sale marchandise !... » (Ghelderode, 1978 : 62) L'homme y arrive « avec une boule énorme à la place du crâne... C'était Smet !... Ses linges semblaient infects, noirâtres... Mais voilà, deux jours de bombardement, un jour de barrage à l'arrière de la ligne, un jour d'attente, et un nouveau jour pour arriver ici...» (Ghelderode, 1978: 64) Les deux amis qui déambulent dans le cimetière s'écartent de la cérémonie au moment où " une cabotine fardée et en grand deuil grimp[e] sur le tertre, et bêlant comme une chèvre à chaque rime, récit[e] des vers pompiers d'un poète officiel.» (Ghelderode, 1978: 67) Les deux amis croisent alors un couple enlacé. L'homme qui les regarde est celui que l'on est censé enterrer. L'hallucination propre au fantastique réel n'est donc pas totalement absente de ces textes de jeunesse. Elle s'y allie à un mélange de trivial et de dénigrement. Elle s'inscrira plus clairement, vingt ans plus tard, dans les proses fantastiques de Sortilèges (1941) dont la composition s'effectue durant les années-clés 1939-1940. L'édition définitive de 1947 est préfacée par Franz Hellens. Hellens est notamment frappé par la nouvelle Un crépuscule qui se déroule dans une ville apparemment vide, et comme en ruines, où les boues cernent l'église Saint-Nicolas dont l'état « était surtout propre à éveiller l'idée du péril » (Ghelderode, $1982: 135)^{24}$.

41 Les années d'occupation ont donc été tout sauf neutres pour plusieurs écrivains nouveaux, qui allaient marquer de leurs empreintes respectives l'Entre-deux-guerres, et qui réagirent au double séisme du conflit mondial et de la révolution sociale par la recherche de Formes novatrices. D'autres, qui eurent à affronter l'épreuve du feu et publièrent donc dans de tout autres conditions, chercheront à réhabiter une idée de l'Homme et du Pays, qui ne passait pas forcément par des recherches déconstructivistes.

\section{BIBLIOGRAPHIE}

BEYEN, Roland (1971). Michel de Ghelderode ou La Hantise du masque. Bruxelles : Académie royale de Langue et de Littérature françaises.

Costenoble, Philostène [pseud. de Michel de Ghelderode] (1928). « Tout est fini... », in Ixelles, mes amours... Poèmes (1924-1927). Ostende : Cahiers de La Flandre Littéraire (L'Aquarium).

DE SCHAEPDRIJVE, Sophie (2008). « Antimémoire d'une antimémoire. Les occupations de l'écrivain belge Georges Eekhoud », in Annamaria Laserra, Nicole Leclercq et Marc Quaghebeur (dir.). Mémoires et Antimémoires littéraires au $\mathrm{XX}^{e}$ siècle. La Première Guerre mondiale. Bruxelles : P.I.E. Peter Lang (Documents pour l'Histoire des Francophonies ; 15), pp. 19-32.

GHELDERODE, Michel de (1978). L'Homme sous l'uniforme. Bruxelles : Louis Musin. GHELDERODE, Michel de Ghelderode (1982). Sortilèges. Bruxelles : Éditions Jacques Antoine (Passé Présent). 
GHELDERODE, Michel de voir aussi cOSTENOBLE, Philostène

MICHEL, Geneviève (2011). Paul Nougé. La poésie au cœur de la révolution. Bruxelles : P.I.E. Peter Lang (Documents pour l'Histoire des Francophonies. Série Europe).

PANSAERS Clément (1921). « Dada et Moi », in Ça ira!, n 16, in Ça ira ! Collection complète 1920-1923

[en fac-similé] (1973). Bruxelles : Éditions Jacques Antoine.

PLISNIER Charles (1921). Réformisme ou Révolution. Anvers : Ça ira!

QUAGHEBEUR Marc (1982). « Balises pour l'histoire de nos lettres », in Alberte Spinette (éd.),

Alphabet des lettres belges de langue française. Bruxelles : Association pour la Promotion des lettres

belges de langue française, pp. 65-70.

Résurrection. Cahiers mensuels littéraires illustrés. Collection complète 1917-1918 (1973). Bruxelles :

Éditions Jacques Antoine.

ROLAND, Hubert (2003). La «Colonie » littéraire allemande en Belgique. 1914-1918. Bruxelles : Labor

(Archives du futur).

\section{NOTES}

1. Ces journaux demeurent inédits. Sophie De Schaepdrijver s'est intéressée à certains aspects de celui d'Eekhoud (De Schaepdrijver, 2008 : 19-32). Le sujet demeure largement en friche même si le Journal informatique de la guerre, dont les AML ont entamé la publication en juillet 2014 ( http://1418.aml-cfwb.be/), en donne certains fragments.

2. Hubert Roland y a consacré un livre : La "Colonie» littéraire allemande en Belgique. 1914-1918 (Roland, 2003). Il y détaille entre autres la politique de traduction allemande d'auteurs d'origine flamande, dont Georges Eekhoud ou Stijn Streuvels bénéficièrent chez Insel Verlag, maison qui publie aussi le mensuel Der Belfried. Eine Monatsschrift für Gegenwart und Geschicht der belgischen Land (juillet 1916-décembre 1918). Cette politique allait de pair avec la Flamenpolitik. L'analyste fournit en outre de précieuses informations sur certains dessous de l'aventure de Résurrection, revue animée par Clément Pansaers.

3. Son nom est annoncé pour le $4^{\mathrm{e}}$ numéro de Résurrection dont il sera question dans les pages qui suivent Il n'y figure toutefois pas.

4. Toutes les références à la revue Résurrection sont indiquées de la sorte : nom de l'auteur, titre de la revue, numéro de la revue, année: page, celle-ci correspondant à la pagination de la réédition de 1973.

5. Pansaers considère que la France assimile l'artistique "par la simplicité », qualification qui n'est pas sans renvoyer à la doxa de la langue française mise en place de longue date, d'une part, et, de l'autre, à ce que paraît rechercher cette génération - on l'a repéré chez Michaux, par exemple.

6. Le poème de Raoul Ravache, qui ouvre le numéro 3 de la revue, débute par : «L'année nouvelle très doucement s'est avancée / Sans une tâche et blanche de neige, / Et mon âme s'est renouvelée, / Vois-tu, pour mieux t'aimer. » (Ravache, Résurrection, 3, $1918: 81$ )

7. C'est moi qui souligne. Jamais Nougé n'eût pu écrire ou envisager cette pensée.

8. Hubert Roland signale par exemple le souci qu'avait Pansaers de publier sa revue à Vienne ou à Cologne, lieux bien plus prestigieux que Namur et qui évitaient la mention berlinoise. Franz Pfemfert, directeur de la revue Die Aktion (Pansaers la loue dans le second volet de son étude de la jeune littérature allemande), signale à Sternheim l'impossibilité de ce dessein. Avec des termes qui ne trompent pas: "'Monsieur Pansaers est-il si naïf qu'il ne reconnaît pas son rôle ?'" (Roland, 2003 : 190) Un rapport d'activité de Von der Lancken, le responsable de la Politische 
Abteilung en Belgique occupée (janvier 1918) mentionne clairement le fait que la revue «"ouvre la voie à une séparation politique de la Flandre et de la Wallonie, par exemple, sous la forme d'une transformation de la Belgique en un État fédéral, consistant en deux États indépendants." " (Roland, 2003: 184); et que la désignation par le II ${ }^{\mathrm{e}}$ Reich de deux gouvernements distincts, lesquels n'ont revêtu aucune légitimité pour la presque totalité des Belges (ce qui n'est sans doute pas le cas de Pansaers), revêt peut-être une certaine importance dans la compréhension historique sur le long terme.

9. Dans son ouvrage italien de 1916, Jules Destrée a avoué lui aussi sa prise en considération de celle-ci. Le Roi le fait explicitement en novembre 1918. Les conséquences ne sont pas les mêmes pour autant.

10. «Les révolutions de l'est de l'Europe nous ont donné des exemples, jusqu'à l'excès, que la réalisation d'un idéal a toujours besoin de discipline et quelquefois de la force.» (Pansaers, Résurrection, 5, 1918 : 197).C'est moi qui souligne.

11. La ponctuation dans la citation est celle du texte d'origine.

12. Ce sera aussi celui des rares Wallons proches de l'occupant. Ils écrivent dans L'Avenir wallon.

13. L'Allemagne, dont les positions officielles ont varié, comptait bien, si faire se pouvait, démanteler la Belgique. La figure de la Belgique martyre existait toutefois. Berlin, dans ses propositions de paix, ne pouvait pas ne pas en tenir compte. Les propositions de paix qui firent suite, fin 1916, à la prise de Bucarest en témoignent comme celles de 1917 que Pansaers relaie ouvertement dans le premier numéro de Résurrection.

14. C'est moi qui souligne. Ces deux adjectifs sont souvent ceux que l'on retient pour définir la singularité belge.

15. On retrouvera de cela chez Ghelderode, mais hors dimension sociale.

16. À Bruxelles, des soldats allemands s'en prennent déjà à leurs officiers. Pansaers y assiste. À Berlin, cette tendance révolutionnaire sera écrasée dans le sang.

17. Pansaers ne semble pas se poser la question des conséquences de la révolution russe à Berlin. Dans un second temps, ses propos ont dû inquiéter les services de l'empire qui soutenaient, d'une façon ou d'une autre, Résurrection.

18. Cfr Michel, Geneviève. Paul Nougé. La poésie au cœur de la révolution (Michel, 2011).

19. Dans « Dada et Moi », Pansaers ridiculise la Belgique littéraire, exception faite de lui-même et de Paul Neuhuys qui accueille sa signature.

20. «Chanson du trépané ».

21. C'est moi qui souligne.

22. Le livre débute par un récit de démobilisation : « (...) nul qui ne se sentit pris par une indicible et heureuse angoisse. Ils laissaient mourir en eux le vieil homme. Et cependant, ils s'en souviendraient, les malheureux, de leur équipée. L'uniforme, c'est comme le baiser d'une mauvaise femme : on peut ne plus en vouloir, mais on y resonge parfois. » (Ghelderode, $1978: 15$ )

23. Elles ont sans doute contribué aux réactions littéraires des années 1920-1930 dont j'ai parlé dans les pages qui précèdent.

24. Ce décor, qui n'est pas sans rappeler La Fin de Nieuport d'Hellens voit se dresser une église "qui subsistait comme un vieux corps décalcifié» (Ghelderode, 1982: 136). Le narrateur y pénètre, croit heurter quelqu'un qui s'avère être un «Christ effondré » (Ghelderode, 1982 : 136), à l'instar de ce qu'a évoqué Verhaeren. Il est convaincu de mourir dans cet effondrement/ enlisement mais voit pendre une corde qu'il agrippe. Une cloche sonne, raffermissant le lieu et l'individu: "Cela se fit angéliquement, hors mon intervention. Un gong sonnait l'heure d'un mystère. Seul, un poing surnaturel avait pu frapper l'heure d'or, sur le gouffre du Temps... » (Ghelderode, 1982 : 137) La vie reprend donc mais dans la nuit. L'église est cernée de troupeaux. «C'était leur nuit d'holocauste. (...) Le monde ne finissait pas ; le monde odorait charnellement, après le déluge. Et j'allai avec les troupeaux chantants et si fatalement beaux, sous les projecteurs lunaires, déporté vers les abattoirs cruels où sont sacrifiées les bêtes, dont le sang coule à 
torrents pour apaiser, on ne sait, la colère des dieux, ou la faim des hommes... » (Ghelderode, $1982: 138)$

\section{RÉSUMÉS}

L'occupation allemande de la presque totalité du territoire métropolitain de la Belgique entre 1914 et 1918 met les jeunes écrivains dans des positions bien différentes (Front de l'Yser, d'Afrique ou de Russie ; camps allemands ; pays occupé ; vie en France ou en Grande-Bretagne ; camps hollandais). La révolution russe les y atteint dans ces situations bien différentes. Cet article s'attache aux attitudes de quelques-uns des porteurs de la novation esthétique de l'Entredeux-guerres, particulièrement de Michel de Ghelderode ou de Clément Pansaers. Celui-ci offrit à Ghelderode un premier tremplin littéraire au sein de Résurrection, revue publiée sous le contrôle et avec l'appui de l'occupant, dans laquelle Pansaers tenait une chronique politique analysée dans cette étude. Défenseurs d'idéaux révolutionnaires, Plisnier, Seuphor ou Nougé se taisent, eux, jusqu'à l'armistice de novembre 1918 et n'offrent pas le flanc à l'ambiguïté des comportements et des discours.

The German occupation of almost the whole of the metropolitan territory of Belgium between 1914 and 1918 places young writers in very different positions (Yser Front, African Front or Russian Front; German camps; occupied country; life in France or in Great Britain; Dutch camps). The Russian revolution affected them in these very different situations. This article examines the attitudes of some of the bearers of the aesthetic innovation of the Interwar period, particularly of Michel de Ghelderode or Clément Pansaers. The latter provided Ghelderode with an initial literary springboard in Résurrection, a review published under the control, and with the support, of the occupier, in which Pansaers kept a political chronical which is analysed in this study. As defendants of revolutionary ideas, Plisnier, Seuphor or Nougé, for their part, remained silent until the armistice of November 1918 and never exposed themselves to the ambiguity of behaviours and discourses.

\section{INDEX}

Keywords : World War I, Russian Revolution, avant-garde, pacifism, Collaboration

Mots-clés : Première Guerre mondiale, Révolution Russe, avant-garde, pacifisme, collaboration

\section{AUTEUR}

MARC QUAGHEBEUR

Archives \& Musée de la Littérature (Bruxelles)

marc.quaghebeur@aml-cfwb.be 